\title{
TILING A SQUARE WITH SIMILAR RECTANGLES
}

\author{
C. Freiling And D. Rinne
}

\begin{abstract}
In 1903 M. Dehn proved that a rectangle can be tiled (or partitioned) into finitely many squares if and only if the ratio of its base and height is rational. In this article we show that a square can be tiled with finitely many similar rectangles of eccentricity $r$ if and only if $r$ is an algebraic number and each of its conjugate roots has positive real part.
\end{abstract}

\section{Introduction}

In 1900 Max Dehn [5] proved the following.

Theorem 1. $(\text { Dehn })^{1}$ A regular tetrahedron cannot be cut into a finite collection of polyhedra and then reassembled to form a cube of the same volume.

This solved the third problem on Hilbert's famous list presented to the International Congress of Mathematics on August 8 of the same year. The theorem demonstrates, for example, why the formula for the volume of a pyramid cannot be derived without using some sort of limit process, answering a question which goes back to Gauss (For more on the history of this problem and an excellent exposition of its solution see [2]).

Three years later, Dehn proved the following similar result [6].

Theorem 2. (Dehn) A rectangle can be tiled using finitely many squares if and only if the ratio of its side lengths is a rational number.

Although the statement of this theorem is what one expects, the proof is surprisingly difficult. Dehn's idea was to create a system of linear equations representing each such tiling and then show that the system has a unique solution. If the rectangle is assumed to have base 1 then the sides of all the square tiles, and hence also the height of the rectangle, must be rational numbers. Dehn actually proved a more general result which we will state and prove in Section 4.

Key words and phrases. Eccentricity, Hamel basis, tiling, partition.

Received May 11, 1994

Both authors are partially supported by NSF.

${ }^{1}$ This theorem remains true even if we are allowed to reverse the orientation of the pieces and even if we are allowed to first add congruent polyhedra to the tetrahedron and cube. 
In a seemingly unrelated development, Hamel (1905 [13]) showed that the Axiom of Choice implies the existence of a non-linear function satisfying Cauchy's functional equation $f(x+y)=f(x)+f(y)$. The construction uses what is now referred to as a Hamel basis, that is, a basis for the vector space of real numbers over the field of rationals. Since the dimension of this space is infinite (in fact the same size as the continuum) the existence of such a basis relies on some form of the Axiom of Choice. Once such a basis is assumed, the construction of a Hamel function is easy. The function $f$ is not only non-linear, but can be assigned arbitrary values on a linearly independent set as follows. Given a linearly independent set, extend it if necessary, to a Hamel basis $H$. For each $h \in H$ choose $f(h)$ arbitrarily. Any real number $x$ can be uniquely expressed as $a_{1} h_{1}+\cdots+a_{n} h_{n}$ where each $a_{i}$ is a nonzero rational number and each $h_{i} \in H$. Define $f(x)=a_{1} f\left(h_{1}\right)+\cdots+a_{n} f\left(h_{n}\right)$. The additivity of $f$ is then easily checked.

Dehn's original proofs of Theorems 1 and 2 are complicated and improvements have been made over the years. In 1940, Brooks, Smith, Stone, and Tutte [4] gave a creative new way to conceptualize Theorem 2. They showed how to transform a square partition of a rectangle into an electric circuit by using Kirchoff's Laws. Each square in the tiling is represented by a wire with unit resistance and the current through each wire represents the side length of the square. The nodes of the circuit represent the horizontal line segments of the tiling, and the wires connect the nodes the same way the squares connect the horizontal line segments. Thus the voltage drop for the entire circuit is the height of the tiled rectangle. By using the fact that Kirchoff's Laws are satisfied (as long as the tiles are squares) and that these laws are sufficient to solve for the currents in each wire (see, for example, [1]) they deduced that the currents must all be rational multiples of the voltage drop. This translates into the sides of the squares (and therefore also the base of the original rectangle) being rational multiples of the height of the rectangle.

Reproving Dehn's theorem was not their only motivation. They applied this technique to help find a tiling of a square into 26 other squares, each of a different size, disproving a conjecture of Lusin. Such "perfect" squarings have also been discovered by others. See [14] for a survey.

It was not until the 1950's that the Swiss geometer Hadwiger and his students connected the ideas of Hamel to the solution of Hilbert's third problem (see [9],[10],[11]). Using a Hamel function, they created a notion of volume. Like usual volume, this new measurement is preserved when polyhedra are cut into other polyhedra and moved around. The proof is then completed by showing that the new volume of the regular tetrahedron is positive while that of the cube is zero. As Boltianskii pointed out in [2] and [3], although the Axiom of Choice is implicitly used in such an 
argument, it can in fact be easily dispensed with.

Hamel functions were also used by Hadwiger [12] to give a transparent proof of Theorem 2. (Also see Pokrovskii [16].) This proof is the primary inspiration behind our main result. We therefore, as an introduction to the techniques, provide this proof in detail in Section 3.

\section{Notation and definitions}

We consider rectangles oriented in the plane with sides parallel to the coordinate axes. By a $b \times h$ rectangle we mean one with base $b$ and height $h$. The eccentricity of a $b \times h$ rectangle is $\max \left(\frac{h}{b}, \frac{b}{h}\right)$. We use $\mathbb{Q}$ and $\mathbb{R}$ to denote the sets of rational numbers and real numbers respectively and $\mathbb{Q}\left[x_{1}, \ldots, x_{n}\right]$ is the ring of polynomials in the variables $x_{1}, \ldots, x_{n}$ with coefficients in $\mathbb{Q}$. For a matrix $A$, we use $A^{\prime}$ for the transpose of $A$.

\section{Hadwiger/Pokrovskii proof of Theorem 2}

Let $R$ be a $b \times h$ rectangle of eccentricity $r$. We may assume that $h \geq b$. If $r=\frac{h}{b}=\frac{p}{q} \in \mathbb{Q}$ where $p$ and $q$ are integers then $R$ can be easily partitioned by $p q$ squares, each being $\frac{b}{q} \times \frac{b}{q}$.

Suppose then that $r$ is irrational. Then $h$ and $b$ are linearly independent over $\mathbb{Q}$. Let $f$ be a Hamel function with $f(b)=1$ and $f(h)=-1$. Define the Hamel area of any $x \times y$ rectangle to be $f(x) f(y)$. The Hamel area of $R$ is then -1 while the Hamel area of any square is nonnegative. The proof is completed by showing that if $R$ is partitioned into subrectangles, the area of $R$ is the sum of the areas of the partitioning rectangles. In other words, this area function is additive. Let $R^{\prime}=R_{1} \cup R_{2}$ be rectangles as in Fig. 1.

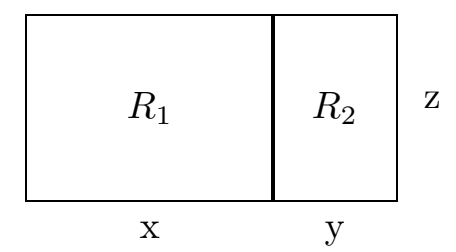

Figure 1

Then the Hamel area of $R^{\prime}$ is $f(x+y) f(z)=f(x) f(z)+f(y) f(z)$ which is simply the Hamel area of $R_{1}$ plus the Hamel area of $R_{2}$, so we have this additive property for Hamel area. Note that any partition of $R$ into rectangles has a refinement which forms a grid. (see Fig. 2) 


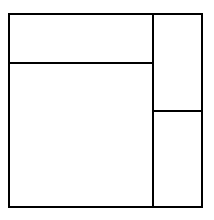

refined to

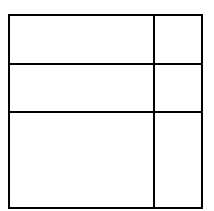

Figure 2

It follows from the additive property above that the Hamel area of $R$ is just the sum of the Hamel areas of the partitioning rectangles (since both are the sum of the areas of the partitioning rectangles in the refinement).

By varying the definition of Hamel area, one easily obtains generalizations which do not seem to lend themselves to the Kirchoff's Law approach. For example, suppose we have rectangular tiles in two colors, red and antired. If a red tile overlaps an anti-red tile, the two colors "cancel" in the overlap and become transparent. If two red tiles overlap, they form a dark red region that could be cancelled by two anti-red tiles stacked on top of them. Three red tiles stacked up form a dark dark red region, and so on. Now consider our irrational rectangle sitting in a white plane. Can this rectangle be covered with red and anti-red tiles so that it appears uniform in color (but not white) while the complement of the rectangle appears to remain white? The answer is no, but the Hamel area proof above fails since, once we allow squares to be subtracted as well as added, arriving at a negative overall Hamel area is no longer a contradiction as desired.

However, the following modification can be made (also see [15]). Let us redefine Hamel area for an $x \times y$ rectangle to be $x f(y)-y f(x)$. This is a slightly stranger definition of area since changing the orientation of a rectangle without changing size causes a sign change in its Hamel area. Nonetheless, it is easy to see that the crucial additive property of Hamel area is preserved. The proof of the red/anti-red problem is then finished by noting that the Hamel area of any square is zero, while the Hamel area of $R$ is $b f(h)-h f(b)=-b-h \neq 0$.

\section{Main results}

The following theorem of Dehn, given here using a Hamel basis type proof, will be used later and relates the eccentricity of a rectangle to the eccentricities of partitioning subrectangles. Observe that this theorem implies one direction of Theorem 2.

Theorem 3. (Dehn) Let $R_{1}, \ldots, R_{n}$ be a partition of the rectangle $R_{0}$, with bases $b_{i}$ and heights $h_{i}, 0 \leq i \leq n$. If $r_{i}=h_{i} / b_{i}$, then $r_{0}$ is a rational function of $r_{1}, \ldots, r_{n}$ with rational coefficients. 
Proof. Let $P$ be the field of rational functions using polynomials in $\mathbb{Q}\left[r_{1}, \ldots, r_{n}\right]$ and consider the reals as a vector space over $P$. If $r_{0} \notin P$, we may select a basis using $b_{0}$ and $h_{0}$ as the first two basis elements. Define the Hamel area of a $b \times h$ rectangle to be $\Gamma_{1}(b) \Gamma_{2}(h)-\Gamma_{1}(h) \Gamma_{2}(b)$ where $\Gamma_{1}(r)$ and $\Gamma_{2}(r)$ are the coefficients of $b_{0}$ and $h_{0}$ respectively in the basis representation of $r$. Then the area of $R_{0}$ is $\Gamma_{1}\left(b_{0}\right) \Gamma_{2}\left(h_{0}\right)-\Gamma_{1}\left(h_{0}\right) \Gamma_{2}\left(b_{0}\right)$ which is $1 \cdot 1-0 \cdot 0=1$ while the area of each subrectangle is $\Gamma_{1}\left(b_{i}\right) \Gamma_{2}\left(h_{i}\right)$ $\Gamma_{1}\left(h_{i}\right) \Gamma_{2}\left(b_{i}\right)$ which is $\Gamma_{1}\left(b_{i}\right) \Gamma_{2}\left(r_{i} b_{i}\right)-\Gamma_{1}\left(r_{i} b_{i}\right) \Gamma_{2}\left(b_{i}\right)=r_{i}\left[\Gamma_{1}\left(b_{i}\right) \Gamma_{2}\left(b_{i}\right)-\right.$ $\left.\Gamma_{1}\left(b_{i}\right) \Gamma_{2}\left(b_{i}\right)\right]=0$. It is easy to see that this area has the desired additive property, and this gives a contradiction. Thus $r_{0} \in P$.

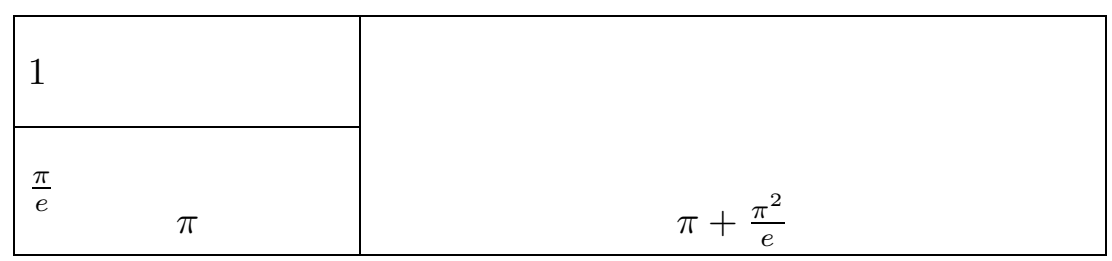

Figure 3

A simple example shows (see Fig. 3) that $r_{0}$ need not be in $\mathbb{Q}\left[r_{1}, \ldots, r_{n}\right]$. The rectangle partitioned by subrectangles of eccentricities $\pi$ and $e$ has $r_{0}=\frac{2 \pi e+\pi^{2}}{e+\pi}$.

In fact, the rational function in Theorem 3 takes on a special form, as the next lemma shows.

Lemma 4. Let $R_{1}, \ldots, R_{n}$ be a partition of the rectangle $R_{0}$ as in Theorem 3. Then $r_{0}=\frac{P\left(r_{1}, \ldots, r_{n}\right)}{Q\left(r_{1}, \ldots, r_{n}\right)}$ where $P$ and $Q$ are polynomials with rational coefficients, all terms of $P$ are of the same degree, all terms of $Q$ are of the same degree, and the degree of $Q$ is one less than the degree of $P$.

Proof. We know that $r_{0}$ is a ratio of two polynomials in $\mathbb{Q}\left[r_{1}, \ldots, r_{n}\right]$ by Theorem 3. Let $\eta$ be any number which is transcendental over $r_{1}, \ldots, r_{n}$. Scaling the vertical axis by a factor of $\eta$ gives a rectangle of eccentricity $\eta r_{0}$ which is partitioned by subrectangles with height-to-width ratios $\eta r_{1}, \ldots, \eta r_{n}$. Using Theorem 3 again, we have $\eta r_{0}=\frac{P^{\prime}\left(\eta r_{1}, \ldots, \eta r_{n}\right)}{Q^{\prime}\left(\eta r_{1}, \ldots, \eta r_{n}\right)}$ for some polynomials $P^{\prime}$ and $Q^{\prime}$. Thus $\eta \frac{P\left(r_{1}, \ldots, r_{n}\right)}{Q\left(r_{1}, \ldots, r_{n}\right)}=\frac{P^{\prime}\left(\eta r_{1}, \ldots, \eta r_{n}\right)}{Q^{\prime}\left(\eta r_{1}, \ldots, \eta r_{n}\right)}$ so

$$
\eta P\left(r_{1}, \ldots, r_{n}\right) Q^{\prime}\left(\eta r_{1}, \ldots, \eta r_{n}\right)=P^{\prime}\left(\eta r_{1}, \ldots, \eta r_{n}\right) Q\left(r_{1}, \ldots, r_{n}\right) .
$$

Now let $A\left(r_{1}, \ldots, r_{n}\right)$ be the sum of the terms of largest degree, say degree $k$, in $P\left(r_{1}, \ldots, r_{n}\right)$, and let $B\left(r_{1}, \ldots, r_{n}\right)$ be the corresponding sum for $Q\left(r_{1}, \ldots, r_{n}\right)$, say of degree $l$. Then the leading term of $P\left(\eta r_{1}, \ldots, \eta r_{n}\right)$ (considered as a polynomial in $\eta$ ) is $A\left(r_{1}, \ldots, r_{n}\right) \eta^{k}$ and the leading term of 
$Q\left(\eta r_{1}, \ldots, \eta r_{n}\right)$ is $B\left(r_{1}, \ldots, r_{n}\right) \eta^{l}$. This says that the leading term on the left side of equation (1) is $\eta P\left(r_{1}, \ldots, r_{n}\right) B\left(r_{1}, \ldots, r_{n}\right) \eta^{l}$ while the leading term on the right side is $Q\left(r_{1}, \ldots, r_{n}\right) A\left(r_{1}, \ldots, r_{n}\right) \eta^{k}$. Since $\eta$ is transcendental over $r_{1}, \ldots, r_{n}$, these terms must be equal so $k=l+1$ and $\frac{P\left(r_{1}, \ldots, r_{n}\right)}{Q\left(r_{1}, \ldots, r_{n}\right)}=\frac{A\left(r_{1}, \ldots, r_{n}\right)}{B\left(r_{1}, \ldots, r_{n}\right)}$ as desired.

Lemma 4 leads to the following result which rules out using a transcendental eccentricity to partition a square.

Lemma 5. If a square is partitioned by rectangles of eccentricity $\eta$ then $\eta$ is algebraic.

Proof. By the previous lemma $1=\frac{P(\eta, 1 / \eta)}{Q(\eta, 1 / \eta)}$ where each term in $P$ is of the form $a_{i} \eta^{i}\left(\frac{1}{\eta}\right)^{k-i}=a_{i} \eta^{2 i-k}$, and each term of $Q$ is of the form $b_{i} \eta^{i}\left(\frac{1}{\eta}\right)^{l-i}=b_{i} \eta^{2 i-l}$ where $k=l+1$. So, the powers of $\eta$ in the terms of $P$ are all of the same parity and the powers in $Q$ are all of the opposite parity. This says that $\eta^{k}[Q(\eta, 1 / \eta)-P(\eta, 1 / \eta)]$ represents a nontrivial polynomial with root $\eta$.

The next two lemmas will be used in the proof of our main result.

Lemma 6. If $p(x) \in \mathbb{Q}[x]$ is irreducible and has $\pm r$ as nonzero roots, then $p$ is even.

Proof. Let $E(x)$ and $D(x)$ be the polynomials formed by the even and odd power terms of $p(x)$ respectively. Then $E(-r)=E(r)$ and $p(-r)=p(r)=$ 0 so $D(-r)=p(-r)-E(-r)=p(r)-E(r)=D(r)$. But $D(-r)=-D(r)$ since $D$ is odd. Thus $D(r)=0$ and this also gives $E(r)=0$. If $D$ is not identically zero, then $D(x)=x F(x)$ where $F$ is of degree strictly smaller than that of $p$. Then $F(r)=0$ so $F$ is not relatively prime to $p$, contradicting the fact that $p$ is irreducible. Thus $p=E$ as desired.

Corollary 7. Let $r>0$ be algebraic with minimal polynomial $p(x)$. Then all roots of $p$ have positive real part or $p$ has a root with strictly negative real part.

Proof. If the roots of $p$ do not all have positive real part then either $p$ has a root with strictly negative real part, in which case we are done, or else $p$ has pure imaginary roots $\pm b i$. Then by the previous lemma, $p$ is an even polynomial. Since $p$ has the real root $r>0,-r<0$ is also a root and we are done.

Lemma 8. If $p(x) \in \mathbb{Q}[x]$ is irreducible then $p$ has distinct roots. 
Proof. If $r$ is a double root of $p$ then it is also a root of the derivative $p^{\prime}$ which is of smaller degree. This says that $p$ and $p^{\prime}$ are not relatively prime contradicting the irreducibility of $p$.

The following theorem of Wall [19] is also used in the proof of our main theorem. See [7] and [18] for a generalization to complex coefficients.

Theorem 9. $(\text { Wall })^{2}$ Let $P(x)=x^{n}+p_{n-1} x^{n-1}+\cdots+p_{0}$ and let $Q(x)=$ $p_{n-1} x^{n-1}+p_{n-3} x^{n-3}+\cdots$ be the alternant of $P(x)$. All roots of $P(x)$ have positive real part if and only if

$$
\frac{Q(x)}{P(x)-Q(x)}=\frac{-1}{c_{n} x+\frac{1}{c_{n-1} x+\frac{1}{\ddots \quad+\frac{1}{c_{1} x}}}}
$$

where each $c_{i}>0$.

We are now ready for the main result.

Theorem 10. ${ }^{3}$ A square can be partitioned using rectangles of eccentricity $r$ if and only if $r$ is algebraic and all the conjugate roots of $r$ have positive real part.

Proof. We first show that $r$ algebraic and having conjugate roots with positive real parts is sufficient to partition a square. Let $P$ be the minimal polynomial for $r$ over the rationals of degree $n$. Using $Q$, the alternant in Wall's Theorem, we have

$$
\frac{Q(r)}{P(r)-Q(r)}=\frac{-1}{c_{n} r+\frac{1}{c_{n-1} r+\frac{1}{\ddots \quad+\frac{1}{c_{1} r}}}}
$$

\footnotetext{
${ }^{2}$ This theorem is usually stated using a polynomial all of whose roots have negative real part. In that case, the numerator of the right side of equation 2 is 1 instead of -1 . Polynomials whose roots have negative real part are important in the stability theory of systems of differential equations. The question of characterizing these polynomials was raised by Maxwell and answered by Routh and Hurwitz (see [8],[17]) using determinants. The continued fraction version due to Wall is more directly applicable to our tiling problem.

${ }^{3}$ This theorem has also been proved independently by M. Laczkovich and G. Szekeres. Their proof will appear in Discrete Geometry, a volume dedicated to the eightieth birthday of L. Fejes-Tóth.
} 
where each $c_{i}>0$. Since $P(r)=0$, we also have $P(r)-Q(r)=-Q(r)$ so $1=\frac{-Q(r)}{P(r)-Q(r)}$. This gives

$$
1=\frac{1}{c_{n} r+\frac{1}{c_{n-1} r+\frac{1}{\ddots \quad+\frac{1}{c_{1} r}}}} .
$$

It remains to show that the right side of the last equality is the ratio of height to base of some rectangle partitioned by rectangles of eccentricity $r$. This will, of course, be a square. We define rectangles $C_{k}$ inductively and show that the corresponding ratio of height to base is

$$
\frac{1}{c_{k} r+\frac{1}{c_{k-1} r+\frac{1}{\ddots \quad+\frac{1}{c_{1} r}}}}
$$

In addition, each $C_{k}$ can be partitioned by rectangles of eccentricity $r$. Let $C_{1}$ be a $c_{1} r \times 1$ rectangle. Then $\frac{1}{c_{1} r}$ is of the desired form. Observe that the ratio of height to base for $C_{1}$ is a rational multiple of $\frac{1}{r}$, so $C_{1}$ can be partitioned using rectangles of eccentricity $r$. Suppose $C_{k}$ has been defined as a $b \times h$ rectangle with

$$
\frac{h}{b}=\frac{1}{c_{k} r+\frac{1}{c_{k-1} r+\frac{1}{\ddots \quad+\frac{1}{c_{1} r}}}}
$$

and that $C_{k}$ can be partitioned using eccentricity $r$. Define $C_{k+1}$ as follows. Rotate $C_{k} 90^{\circ}$ to an $h \times b$ rectangle and adjoin to its right vertical side a $c_{k+1} r b \times b$ rectangle. The resulting $C_{k+1}$ is then $\left(c_{k+1} r b+h\right) \times b$ and the 
ratio of height to base is

$$
\frac{b}{c_{k+1} r b+h}=\frac{1}{c_{k+1} r+\frac{h}{b}}=\frac{1}{c_{k+1} r+\frac{1}{c_{k} r+\frac{1}{\ddots \quad+\frac{1}{c_{1} r}}}}
$$

as desired. Since $C_{k+1}$ is formed by adjoining a partitionable rectangle to $C_{k}$, it is itself partitionable. The rectangle $C_{n}$ is then the desired square.

To prove necessity, suppose that $r$ is algebraic of degree $n$ (we know that $r$ cannot be transcendental by Lemma 5) but has a conjugate root with real part less than or equal to zero. By Corollary 7 we may assume that a conjugate root has negative real part. We will define an area function so that rectangles of eccentricity $r$ have area greater than or equal to zero and construct a square whose area is less than zero. Then this square cannot be partitioned by rectangles of eccentricity $r$. We define our area function via a quadratic form. Since $r$ is algebraic $\mathbb{Q}[r]$ forms a field, the rational polynomials in $r$ of degree less than or equal to $n-1$. Let $H$ be a Hamel basis for $\mathbb{R}$ as a vector space over $\mathbb{Q}[r]$. We may assume that $H$ contains the number 1 . So any element of $\mathbb{R}$ can be uniquely written as $\sum_{i=1}^{k} p_{i}(r) h_{i}$ where $h_{i} \in H$ and each $p_{i}(r) \in \mathbb{Q}[r]$. For $x \in \mathbb{R}$, let $p_{x}(r)$ be the coefficient of 1 in the representation of $x$. We use $[x]$ to denote the usual $n$-tuple representation of $p_{x}(r)$ as a vector in $\mathbb{Q}^{n}$, namely, if $p_{x}(r)=a_{0}+a_{1} r+\cdots+a_{n-1} r^{n-1}$, then $[x]=\left[a_{0}, a_{1}, \ldots, a_{n-1}\right]$. For any $n \times n$ symmetric matrix $M$, we can define the "area" of a $b \times h$ rectangle to be $[b] M[h]^{\prime}$. It is easy to see that this is a valid area function in that it has the desired additive property. Indeed, a $\left(b_{1}+b_{2}\right) \times h$ rectangle has area $\left[b_{1}+b_{2}\right] M[h]^{\prime}=\left[b_{1}\right] M[h]^{\prime}+\left[b_{2}\right] M[h]^{\prime}$. Also, the area of an $h \times b$ rectangle is $[h] M[b]^{\prime}=\left([h] M[b]^{\prime}\right)^{\prime}=[b] M[h]^{\prime}$ since $M$ is symmetric. Since this is also the area of a $b \times h$ rectangle this area function is independent of orientation. Let $p(x)=x^{n}+p_{n-1} x^{n-1}+\cdots+p_{0}$ be the minimal polynomial for $r$ and consider the companion matrix

$$
Q=\left[\begin{array}{ccccc}
0 & 0 & \cdots & 0 & -p_{0} \\
1 & & & \vdots & \\
0 & 1 & & \vdots & \vdots \\
\vdots & & \ddots & 0 & \\
0 & \cdots & 0 & 1 & -p_{n-1}
\end{array}\right]
$$


whose eigenvalues are the roots of $p(x)$. We need to calculate $[r x]$ in terms of $[x]$. If $p_{x}(r)=a_{0}+a_{1} r+\cdots+a_{n-1} r^{n-1}$ then $p_{r x}(r)=r p_{x}(r)=$ $a_{0} r+a_{1} r^{2}+\cdots+a_{n-2} r^{n-1}+a_{n-1} r^{n}$ and solving $p(r)=0$ for $r^{n}$ we get

$$
\begin{aligned}
& p_{r x}(r)= a_{0} r+a_{1} r^{2}+\cdots+a_{n-2} r^{n-1}-a_{n-1}\left(p_{0}+p_{1} r+\cdots+p_{n-1} r^{n-1}\right) \\
&=-a_{n-1} p_{0}+\left(a_{0}-a_{n-1} p_{1}\right) r+\left(a_{1}-a_{n-1} p_{2}\right) r^{2}+\cdots \\
&+\left(a_{n-2}-a_{n-1} p_{n-1}\right) r^{n-1}
\end{aligned}
$$

Then

$$
[r x]^{\prime}=\left[\begin{array}{c}
-a_{n-1} p_{0} \\
a_{0}-a_{n-1} p_{1} \\
\vdots \\
a_{n-2}-a_{n-1} p_{n-1}
\end{array}\right]=Q\left[\begin{array}{c}
a_{0} \\
a_{1} \\
\vdots \\
a_{n-1}
\end{array}\right]=Q[x]^{\prime} .
$$

Thus the area of a $b \times r b$ rectangle will be $[b] M Q[b]^{\prime}$ while a $b \times b$ square will have area $[b] M[b]^{\prime}$. We first consider the case all eigenvalues of $Q$ real. By Lemma 8 the eigenvalues of $Q$ are distinct. Therefore there is an $n \times n$ matrix $P$ that diagonalizes $Q$. That is, $P^{-1} Q P=D$, where $D$ is a diagonal matrix with the eigenvalues of $Q$ (all nonzero) as diagonal entries. By assumption one of the diagonal entries is less than zero. We choose $M$ to be the symmetric matrix $P^{-1 \prime} D P^{-1}$. Using $v=[b] P^{-1 \prime}$, the area of a $b \times r b$ rectangle becomes $[b] M Q[b]^{\prime}=[b] P^{-1 \prime} D P^{-1} Q[b]^{\prime}=v D P^{-1} Q P v^{\prime}=$ $v D^{2} v^{\prime} \geq 0$ for all $b$. Since this area is independent of orientation, we get the same result for any $r b \times b$ rectangle. Without loss of generality, we may assume that the first entry in $D$, say $\lambda_{1}$, is negative. Observe that $[1,0, \ldots, 0] P^{\prime} M P[1,0, \ldots, 0]^{\prime}=[1,0, \ldots, 0] P^{\prime} P^{-1 \prime} D P^{-1} P[1,0, \ldots, 0]^{\prime}=$ $\lambda_{1}<0$. However, the entries of $[1,0, \ldots, 0] P^{\prime}$ may be irrational. Therefore, pick $s$ to be any positive number with $[s]$ sufficiently close to $[1,0, \ldots, 0] P^{\prime}$ (in the Euclidean norm) so that $[s] M[s]^{\prime}<\lambda_{1} / 2<0$. Then any $s \times s$ square cannot be partitioned by rectangles of eccentricity $r$. It follows that no square can be so partitioned.

We now consider the case where not all the conjugate roots are real and modify the construction above. We may choose the matrix $P$ so that $P^{-1} Q P=D$ where $D$ is of the form $\left[\begin{array}{ccc}B_{1} & \cdots & 0 \\ \vdots & \ddots & \vdots \\ 0 & \cdots & B_{k}\end{array}\right]$ and each $B_{i}$ is a $1 \times 1$ block for each real eigenvalue or a $2 \times 2$ block of the form $\left[\begin{array}{cc}\alpha & \beta \\ -\beta & \alpha\end{array}\right]$ for each pair of complex eigenvalues $\alpha \pm i \beta$. Let $\Delta$ be the diagonal matrix whose diagonal entries are the same as those in $D$, that is, the real parts of the eigenvalues of $Q$. We choose $M=P^{-1 \prime} \Delta P^{-1}$. The area of a $b \times r b$ rectangle is then $[b] M Q[b]^{\prime}=[b] P^{-1 \prime} \Delta P^{-1} Q[b]^{\prime}=v \Delta P^{-1} Q P v^{\prime}=$ 
$v \Delta D v^{\prime}=\sum_{i=1}^{n} \alpha_{i}^{2} v_{i}^{2} \geq 0$ where the $v_{i}$ and $\alpha_{i}$ are the components of $v$ and the real parts of the eigenvalues of $Q$ respectively. We again get the same result for any $r b \times b$ rectangle. By Lemma 7 we know that one eigenvalue of $Q$ has negative real part, $\alpha$, and we may assume that this is the first diagonal entry in $\Delta$. Then $[1,0, \ldots, 0] P^{\prime} M P[1,0, \ldots, 0]^{\prime}=$ $[1,0, \ldots, 0] P^{\prime} P^{-1 \prime} \Delta P^{-1} P[1,0, \ldots, 0]^{\prime}=\alpha<0$. By the same argument as above, we may pick an $s \times s$ square with area less than $\alpha / 2$. This square of negative area cannot be partitioned by rectangles of eccentricity $r$. Hence no square can be so partitioned.

We include an example of the kind of partition created in the proof of Theorem 10. The polynomial $P(x)=x^{3}-6 x^{2}+12 x-3$ has three roots on the circle centered at $z=2$ with radius $\sqrt[3]{5}$. One of these is the real number $r=2-\sqrt[3]{5}$. The alternant is $Q(x)=-6 x^{2}-3$ and

$$
\frac{-Q(x)}{P(x)-Q(x)}=\frac{1}{\frac{1}{6} x+\frac{1}{\frac{12}{23} x+\frac{1}{\frac{23}{6} x}}}
$$

We partition a square as illustrated using $c_{1}=\frac{23}{6}, c_{2}=\frac{12}{23}$ and $c_{3}=\frac{1}{6}$.

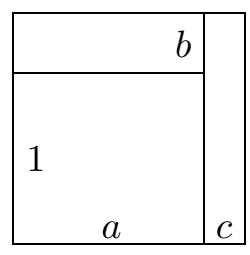

Figure 4

We then have $a=c_{1} r=\frac{23}{6} r, b=c_{2} r a=2 r^{2}$ and $c=c_{3} r(1+b)=$ $\frac{1}{6} r+\frac{1}{3} r^{3}$. This rectangle is then $(a+c) \times(1+b)=\left(4 r+\frac{1}{3} r^{3}\right) \times\left(1+2 r^{2}\right)$. Since $r^{3}-6 r^{2}+12 r-3=0,\left(4 r+\frac{1}{3} r^{3}\right)=\left(1+2 r^{2}\right)$ so this is indeed a square.

The authors would like to thank Noa Goldring and Greg Tollisen of Occidental College for finding and correcting an earlier mistake in our area definition.

\section{References}

1. D. W. Blackett, Elementary Topology: A Combinatorial and Algebraic Approach, Academic Press, New York - London.

2. V. G. Boltianskii, Hilbert's Third Problem (trans. by R. Silverman), V. H. Winston \& Sons, Washington D. C., 1978. 
3. Equivalent and Equidecomposable Figures (trans. by A.K.Henn and C.E. Watts), D. C. Heath and Co., Boston, 1963.

4. R. L. Brooks, C. A. B. Smith, A. H. Stone, and W. T. Tutte, The dissection of rectangles into squares, Duke Math. Journal 7 (1940), 312-340.

5. M. Dehn, Über raumgleiche Polyeder, Nachr. Akad. Wiss. Göttingen Math.-Phys. Kl. (1900), 345-354.

6. _ـ Über die Zerlegung von Rechtecken in Rechtecke, Math. Ann. 57 (1903), 314-332.

7. E. Frank, On the zeros of polynomials with complex coefficients, Bull. Amer. Math. Soc. 52 (1946), 144-157.

8. E. A. Guillemin, The Mathematics of Circuit Analysis, Principles of Electrical Engineering Series, M.I.T. Press, Cambridge, MA, 1949, 9th printing 1969.

9. H. Hadwiger, Zum Problem der Zerlegungsgleichheit der Polyeder, Arch. Math. 2 (1949-50), 441-444.

10. _ Lineare additive Polyederfunctionale und Zerlegungsgleichheit, Math. Zeits. 58 (1953), 4-14.

11. Zum Problem der Zerlegungsgleichheit k-dimensionaler Polyeder, Math. Ann. 127 (1954), 170-174.

12. — Vorlesungen Über Inhalt, Oberfläche und Isoperimetrie, Springer-Verlag, 1957.

13. G. Hamel, Eine Basis aller Zahlen und die unstetigen Lösungen der Funktionalgleichung: $f(x+y)=f(x)+f(y)$, Math. Ann. 60 (1905), 459-462.

14. N. D. Kazarinoff and R. Weitzenkamp, Squaring rectangles and squares, Amer. Math. Monthly 80 (1973), 877-888.

15. R. Kenyon, Tiling with squares and square-tileable surfaces, Preprint, CNRS-URM 128, Lyon, France.

16. V. G. Pokrovskii, Slicings of $n$-dimensional parallelepipeds, trans. from Matematicheskie Zametki 33 No.2 (1983), 273-280.

17. J. V. Uspensky, Theory of Equations, McGraw-Hill, New York, 1948.

18. H. S. Wall, Analytic Theory of Continued Fractions, Chelsea Pub. Co., Bronx, N.Y., 1967, originally The University Series in Higher Mathematics Vol. I, D. Van Nostrand Co., New York, 1948.

19. 52 (1945), 308-322.

California State University, San Bernardino, CA 92407

E-mail address: cfreilin@wiley.csusb.edu, drinne@wiley.csusb.edu 